\title{
Increasing burden of nursing care on the treatment of COVID-19 patients in the ageing society: analyses during the first to the third wave of pandemic in Kyoto City, Japan
}

Kohei Fujita ( $\nabla$ kfujita.acd@gmail.com )

National Hospital Organization Kyoto Medical Center

Eriko Kashihara

Tenri Hospital

Osamu Kanai

National Hospital Organization Kyoto Medical Center

Hiroaki Hata

National Hospital Organization Kyoto Medical Center

Akihiro Yasoda

National Hospital Organization Kyoto Medical Center

Takao Odagaki

National Hospital Organization Kyoto Medical Center

Tadashi Mio

National Hospital Organization Kyoto Medical Center

\section{Research Article}

Keywords: COVID-19, Ageing society, nursing care, elderly patients, healthcare workers

Posted Date: May 14th, 2021

DOI: https://doi.org/10.21203/rs.3.rs-492626/v1

License: (c) (1) This work is licensed under a Creative Commons Attribution 4.0 International License.

Read Full License

Version of Record: A version of this preprint was published at Frontiers in Medicine on November 18th, 2021. See the published version at https://doi.org/10.3389/fmed.2021.767110. 


\section{Abstract \\ Background}

The coronavirus disease 2019 (COVID-19) pandemic is associated with a heavy burden on patients' mental and physical health, regional healthcare resources, and global economic activity. An ageing society such as Japan has many retirement homes and long-term stay hospitals for the elderly and their inhabitants. During the COVID-19 pandemic, disease clusters are often identified in retirement homes and long-term stay hospitals. Although we hypothesize that additional burdens of nursing care for elderly patients will reinforce the anxiety and exhaustion of medical staff and healthcare resources in the ageing society, the actual situation is not well understood. In this study, we aimed to evaluate the current situation and countermeasures of the COVID-19 pandemic in the ageing society.

\section{Methods}

We reviewed COVID-19 patients who required hospitalization at the National Hospital Organization Kyoto Medical Center, a 600-bed capacity hospital located in Kyoto, Japan, between 1 April 2020 and 31 March 2021. We assessed the characteristics of the COVID-19 patients, disease severity, duration of hospitalization, outcome at discharge, degree of activities of daily living (ADLs), and complications unique to elderly patients.

\section{Results}

We enrolled 118 patients who required hospitalization during the study period. Approximately $40 \%$ of the patients were aged $\geq 80$ years. Dementia $(27.1 \%)$ was the most prevalent underlying disease, followed by diabetes mellitus (23.7\%) and chronic kidney disease (23.7\%). Approximately $60 \%$ of hospitalized COVID19 patients had impaired ADL at admission. The COVID-19 patients aged 80 years or older required significantly more longer-term hospitalization than the COVID-19 patients aged under 80 years $(15.5 \pm 8.2$ vs. $13.1 \pm 7.7, P=0.032$ ). In elderly patients aged 80 years or older, approximately $50 \%$ of patients had geriatric mental disorders, and approximately $70 \%$ had bedridden status and feeding difficulty.

\section{Conclusions}

The proportion of elderly patients aged 80 years or older was relatively high during the hospitalization for COVID-19. We should keep in mind that healthcare workers are forced to have an additional burden of nursing care in the ageing society during the COVID-19 pandemic. Therefore, interventions to reduce the burden are urgently required.

\section{Background}


Coronavirus disease 2019 (COVID-19) is caused by severe acute respiratory syndrome coronavirus-2 (SARS-CoV-2). COVID-19 was first identified in Wuhan, China, in December 2019. The outbreak of COVID19 had rapidly spread worldwide, and the World Health Organization declared a pandemic on 11 March 2020. [1] The clinical course of COVID-19 is mild and self-limiting upper respiratory infection symptoms in young healthy subjects; however, severe respiratory failure is often observed in elderly patients with chronic diseases. [2, 3] The COVID-19 pandemic is associated with a heavy burden on patients' mental and physical health, regional healthcare resources, and global economic activity. As is well known, Japan is facing the most ageing society worldwide. [4] There are many retirement homes and long-term stay hospitals for the elderly and their inhabitants in Japan. During the COVID-19 pandemic, disease clusters are often identified in retirement homes and long-term stay hospitals. [5] Elderly patients with chronic diseases had been mainly affected by the COVID-19 pandemic between the first and third waves of the pandemic in Japan. A recent study has shown that clinicians and clinical teams for COVID-19 patient care had struggled with the complexity of providing high-quality care because of the following difficulties: crisis capacity, resource limitation, and multiple unprecedented barriers to care delivery. [6, 7] In addition, healthcare workers had a higher prevalence of stress, anxiety, depression, and psychological distress than the general population during the COVID-19 pandemic. [8]

We hypothesize that additional burdens of nursing care for elderly patients will reinforce the anxiety and exhaustion of medical staff and healthcare resources in the ageing society such as Japan.

In this study, we aimed to evaluate the current situation and countermeasures of the COVID-19 pandemic in the ageing society.

\section{Patients And Methods}

We reviewed COVID-19 patients who required hospitalization at the National Hospital Organization Kyoto Medical Center, a 600-bed capacity hospital located in Kyoto, Japan, between 1 April 2020 and 31 March 2021. During this period, Japan experienced several major outbreaks of COVID-19: the first, second, and third waves of the pandemic. During this pandemic, most tertiary medical centres or hospitals in Kyoto City have accepted mild to severe COVID-19 patients. All COVID-19 patients admitted to our hospital were diagnosed by SARS-CoV-2 PCR tests. Because COVID-19 was designated as a specific infectious disease in Japan, all COVID-19 patients were hospitalized according to the recommendation of public authorities and treated by publicly funded health care. To avoid this concentration, COVID-19 patients living in Kyoto City were assigned to suitable hospitals by the public authorities. The population ageing rate of Kyoto City increased to $28.0 \%$ in 2019, indicating that 1 out of 3.5 people living in Kyoto City was an elderly aged 65 years or older. [9] We assessed the characteristics of COVID-19 patients, disease severity, duration of hospitalization, outcome at discharge, degree of activities of daily living (ADLs), and complications unique to elderly patients. Disease severity was defined as mild, moderate, or severe according to the Japanese COVID-19 treatment guidelines published by the Japanese Ministry of Health, Labour and Welfare. [5] COVID-19 Registry Japan (COVIREGI-JP) of National Center for Global Health and Medicine were used for this study with permission. The study data were collected and managed using 
REDCap (Research Electronic Data Capture) a secure, web-based data capture application hosted at JCRAC data center of National Center for Global Health and Medicine.

\section{Statistics Analyses}

Statistical analyses were performed using the Statistical Package for the Social Sciences (SPSS) version 26 (International Business Machines Corporation SPSS, Chicago, IL, USA). The Wilcoxon rank-sum test was used to compare the duration of hospitalization between the two groups.

\section{Results}

We enrolled 118 patients who required hospitalization during the study period. The characteristics of COVID-19 patients hospitalized in our hospital are shown in Table 1. Patients' mean age was $71.3 \pm 17.4$ years. The female-to-male ratio was roughly half and half. Approximately $40 \%$ of the patients were aged $\geq 80$ years. Dementia (27.1\%) was the most prevalent underlying disease, followed by diabetes mellitus (23.7\%) and chronic kidney disease (23.7\%). The ADLs of the COVID-19 patients are shown in Table 1. Approximately $60 \%$ of hospitalized COVID-19 patients had impaired ADLs at admission. Figure 1 shows age-specific COVID-19 disease severity. Hospitalization was most commonly needed in the elderly in their 70 s, followed by the 80 s and 60 s. Most patients in their 20s and 30 s had mild disease, whereas the majority of patients in their $40 \mathrm{~s}, 50 \mathrm{~s}$, and $60 \mathrm{~s}$ had moderate disease. Furthermore, the vast majority ( $\leq$ $80 \%$ ) of patients aged $\geq 70$ years had moderate and severe diseases. Figure 2 shows the outcomes of COVID-19 patients at discharge. The COVID-19 mortality rate at our hospital was 11\%. All but one patient was in their 70 s to 90 s. Figure 3 shows the duration of the hospitalization. COVID-19 patients aged 80 years or older required significantly more longer-term hospitalization than the COVID-19 patients aged under 80 years $(15.5 \pm 8.2$ vs. $13.1 \pm 7.7, \mathrm{P}=0.032)$. Table 2 shows the complications unique to elderly patients in the COVID-19 care unit. In all patients, approximately $40 \%$ had a bedridden status and feeding difficulty. For elderly patients aged 80 years or older, approximately $50 \%$ of patients had geriatric mental disorder, and approximately $70 \%$ had bedridden status and feeding difficulty. 
Table 1

Characteristics of the coronavirus disease 2019 patients

\begin{tabular}{|ll|}
\hline Characteristics & $\mathbf{N}=118$ \\
\hline Age, years & $71.3 \pm 17.4$ \\
\hline Sex (female) & $61(51.7)$ \\
\hline Elderly patients (aged $\leq 80$ years) & $46(39.0)$ \\
\hline Underlying diseases & $22(18.6)$ \\
\hline Cardiovascular diseases & $12(10.2)$ \\
\hline Cerebrovascular diseases & $32(27.1)$ \\
\hline Dementia & $28(23.7)$ \\
\hline Diabetes mellitus & $17(14.4)$ \\
\hline Chronic pulmonary disease & $28(23.7)$ \\
\hline Chronic kidney disease & $13(11.0)$ \\
\hline Malignant disease & $8(6.8)$ \\
\hline Use of immunosuppressants & $1(0.85)$ \\
\hline Use of biologic agents & $14.1 \pm 8.0$ \\
\hline Duration of hospitalization, days & $48(40.7)$ \\
\hline Activities of daily livings & $41(34.7)$ \\
\hline Total independence & $29(24.6)$ \\
\hline Partial independence & \\
\hline Total dependence & \\
\hline Data were shown as mean \pm standard deviation or number (\%). \\
\hline
\end{tabular}


Table 2

Complications unique to elderly patients

\begin{tabular}{|lll|}
\hline Complications & All patients & Patients aged 80 years or older \\
\cline { 2 - 3 } & $\mathbf{N}=\mathbf{1 1 8}$ & $\mathbf{N}=\mathbf{4 7}$ \\
\hline Bedridden status & $46(39.0)$ & $31(66.0)$ \\
\hline Feeding difficulty & $52(44.1)$ & $34(72.3)$ \\
\hline Enteral alimentation & $19(16.1)$ & $11(23.4)$ \\
\hline Fluid replacement & $33(28.0)$ & $23(48.9)$ \\
\hline Disquiet/delirium & $38(32.2)$ & $26(55.3)$ \\
\hline Dementia/communication difficulty & $40(33.9)$ & $24(51.1)$ \\
\hline Need continuous sputum suction & $27(22.9)$ & $18(38.3)$ \\
\hline Data were shown as number (\%). & & \\
\hline
\end{tabular}

\section{Discussion}

In our hospital, almost $40 \%$ of hospitalized COVID-19 patients were aged 80 years or older. We found that $30-40 \%$ of COVID-19 patients in our hospital experienced complications unique to elderly patients. According to the Japanese government statement, elderly patients aged 80 years or older accounted for $10-15 \%$ of hospitalized COVID-19 patients in Japan. [5] Our hospital had accepted approximately three times higher percentage of elderly COVID-19 patients than the average of other hospitals in Japan. This might be caused by the COVID-19 clusters that occurred in nearby retirement homes and long-term stay hospitals.

Notably, we revealed that only $40 \%$ of the COVID-19 patients in our hospital could maintain their ADLs of total independence at admission. The remaining $60 \%$ of the patients had impaired ADLs. Approximately $30-40 \%$ of all hospitalized patients have some forms of complications unique to elderly patients. In patients aged 80 years or older, the rate of these complications increased by $50-70 \%$. These facts clearly indicate that healthcare workers belonging to COVID-19 care units had a considerable burden of nursing care from the early stages of hospitalization. Furthermore, because our study revealed that elderly patients aged 80 years or older had a significantly longer duration of hospitalization, this situation would exacerbate the bed turnover rate and result in additional physical and mental burden in healthcare workers. Mehta et al. have reported that many healthcare workers are concerned about anxiety, depression, burnout, insomnia, and moral distress under unusual circumstances, which force wearing cumbersome and uncomfortable personal protective equipment and keeping up with emerging knowledge and new technologies of COVID-19. [10] Singh et al.'s systematic review meta-analysis has also shown that healthcare workers experienced a crisis of psychological wellbeing. [8] In addition, our 
study revealed that healthcare workers bear an additional burden of nursing care for elderly patients in the ageing society.

To avoid excessive load and protect healthcare workers from physical and mental stress, several countermeasures should be taken into account during difficult emerging situations such as this pandemic. First, we should consider the importance of early rehabilitation interventions. According to de Biase et al., delivering rehabilitation in the same way as before the COVID-19 pandemic will neither be practical nor meet patients' needs. [11] As mentioned, rehabilitation should be modified as a needs-based, individual approach in response to the COVID-19 pandemic. Careful training of physical therapy for COVID-19 patients is required in each hospital for COVID-19. Second, early approaches for delirium prevention and management must be adapted. The Hospital Elder Life Program provides creative approaches that comprise several intervention methods, such as providing tablet computers for remote coaching and communication by professionals, volunteers, or family members and providing sleep enhancement methods. [12,13] Early interventions for adequate programmes for COVID-19 patients, especially elderly patients, are necessary. Third, both healthcare organizations and healthcare workers should acquire knowledge or skills in stress reduction techniques. Callus et al. have summarized the key interventions for healthcare workers to enhance awareness, self-care interventions, mental health services, digital technologies, and organization approaches. [14] It is important to prepare these key interventions in ordinary times because the rapid implementation of these interventions during the pandemic suggests that safety may not have received sufficient attention. [15] Although the abovementioned methods will require additional work for the initial period of time, they will be of great help to maintain good conditions for both healthcare workers and hospital infrastructure in the long term. There are several limitations in this study. This study had retrospective nature and small sample size. In addition, this study was conducted in a single medical center. These conditions can cause outcome reporting bias and patient selection bias. Because we did not evaluate the burden of nursing care quantitatively, it was difficult to explain the burden in a quantitative manner. Nonetheless, we believe that our study had a significant impact to reveal a burden of nursing care for elderly COVID-19 patients during COVID-19 pandemic.

\section{Conclusion}

In conclusion, we found that the proportion of elderly patients aged 80 years or older was relatively high during the hospitalization for COVID-19. We should keep in mind that healthcare workers are forced to have an additional burden of nursing care in the ageing society during the emerging COVID-19 pandemic. Interventions to reduce the burden for both patients and healthcare workers are urgently needed.

\section{Declarations}

\section{Ethical approval and consent to participate}


This study was approved by the National Hospital Organization Kyoto Medical Center institutional review board (approval number: 20-092). All methods were carried out in accordance with relevant guidelines and regulations. Informed consent was obtained from all patients.

\section{Consent for publication}

Not applicable.

\section{Availability of data and materials}

Data are available from COVIREGI-JP bureau with permission. Only participating institutions can apply for data use.

\section{Competing interests}

The authors declare that they have no competing interests.

\section{Funding}

We received no funds for conducting this study.

\section{Authors' contributions}

$\mathrm{KF}$ and EK drafted and revised the manuscript. $\mathrm{KF}, \mathrm{EK}, \mathrm{OK}$, and $\mathrm{HH}$ collected the data for this study. $\mathrm{HH}$, $A Y, T O$, and TM supervised the study and revised the manuscript. All the authors have approved the manuscript for submission.

\section{Acknowledgments}

We thank all members of the Department of Infectious Disease and Emergency Medicine for their dedicated patient care. COVIREGI-JP of National Center for Global Health and Medicine were used for this study with permission.

\section{References}

1. World Health Organization. World Health Organization COVID-19 Situation Reports. 2021. https://www.who.int/emergencies/diseases/novel-coronavirus-2019/situation-reports. Accessed 28 Apr 2021.

2. Guan W, Ni Z, Hu Y, Liang W, Ou C, He J, et al. Clinical Characteristics of Coronavirus Disease 2019 in China. New England Journal of Medicine. 2020;382:1708-20. doi:10.1056/nejmoa2002032.

3. Chen N, Zhou M, Dong X, Qu J, Gong F, Han Y, et al. Epidemiological and clinical characteristics of 99 cases of 2019 novel coronavirus pneumonia in Wuhan, China: a descriptive study. The Lancet. 
2020;395:507-13. doi:10.1016/S0140-6736(20)30211-7.

4. Statistics Bureau of Japan. Japanese population statistics. 2021. https://www.stat.go.jp/english/index.html. Accessed 28 Apr 2021.

5. Ministry of Health Labour and Welfare of Japan. Coronavirus Diseases Situation in Japan. 2021. https://www.mhlw.go.jp/stf/seisakunitsuite/bunya/0000164708_00079.html. Accessed 28 Apr 2021.

6. Azoulay E, Cariou A, Bruneel F, Demoule A, Kouatchet A, Reuter D, et al. Symptoms of anxiety, depression, and peritraumatic dissociation in critical care clinicians managing patients with COVID19 a cross-sectional study. American Journal of Respiratory and Critical Care Medicine. 2020;202:1388-98. doi:10.1164/rccm.202006-25680C.

7. Butler CR, Wong SPY, Wightman AG, O'Hare AM. US Clinicians' Experiences and Perspectives on Resource Limitation and Patient Care during the COVID-19 Pandemic. JAMA Network Open. 2020;3. doi:10.1001/jamanetworkopen.2020.27315.

8. Singh RK, Bajpai R, Kaswan P. COVID-19 pandemic and psychological wellbeing among health care workers and general population: A systematic-review and meta-analysis of the current evidence from India. Clinical Epidemiology and Global Health. 2021;11. doi:10.1016/j.cegh.2021.100737.

9. Kyoto city. Population Statistics in Kyoto City . 2019. https://www2.city.kyoto.lg.jp/sogo/toukei/Population/Suikei/. Accessed 28 Apr 2021.

10. Mehta S, Machado F, Kwizera A, Papazian L, Moss M, Azoulay É, et al. COVID-19: a heavy toll on health-care workers. The Lancet Respiratory Medicine. 2021;9:226-8. doi:10.1016/S22132600(21)00068-0.

11. de Biase S, Cook L, Skelton DA, Witham M, ten Hove R. The COVID-19 rehabilitation pandemic. Age and Ageing. 2020;49:696-700. doi:10.1093/ageing/afaa118.

12. Inouye SK. The Importance of Delirium and Delirium Prevention in Older Adults during Lockdowns. JAMA - Journal of the American Medical Association. 2021. doi:10.1001/jama.2021.2211.

13. Wang YY, Yue JR, Xie DM, Carter P, Li QL, Gartaganis SL, et al. Effect of the Tailored, Family-Involved Hospital Elder Life Program on Postoperative Delirium and Function in Older Adults: A Randomized Clinical Trial. JAMA Internal Medicine. 2020;180:17-25. doi:10.1001/jamainternmed.2019.4446.

14. Callus E, Bassola B, Fiolo V, Bertoldo EG, Pagliuca S, Lusignani M. Stress Reduction Techniques for Health Care Providers Dealing With Severe Coronavirus Infections (SARS, MERS, and COVID-19): A Rapid Review. Frontiers in Psychology. 2020;11. doi:10.3389/fpsyg.2020.589698.

15. Priede A, López-Álvarez I, Carracedo-Sanchidrián D, González-Blanch C. Mental health interventions for healthcare workers during the first wave of COVID-19 pandemic in Spain. Revista de Psiquiatria y Salud Mental. 2021. doi:10.1016/j.rpsm.2021.01.005.

\section{Figures}


20

10

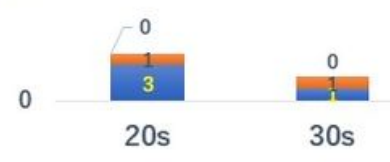

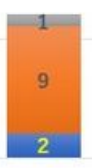

40 s

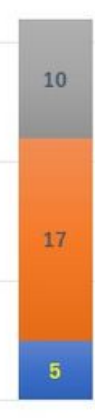

50 s



$60 \mathrm{~s}$

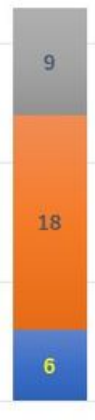

80 s

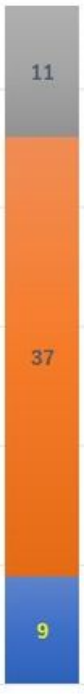

Male

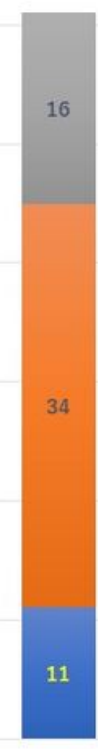

Female

mild moderate a severe

\section{Figure 1}

Age-specific coronavirus disease 2019 disease severity. Blue, red, and grey bars show mild, moderate, and severe disease status, respectively.
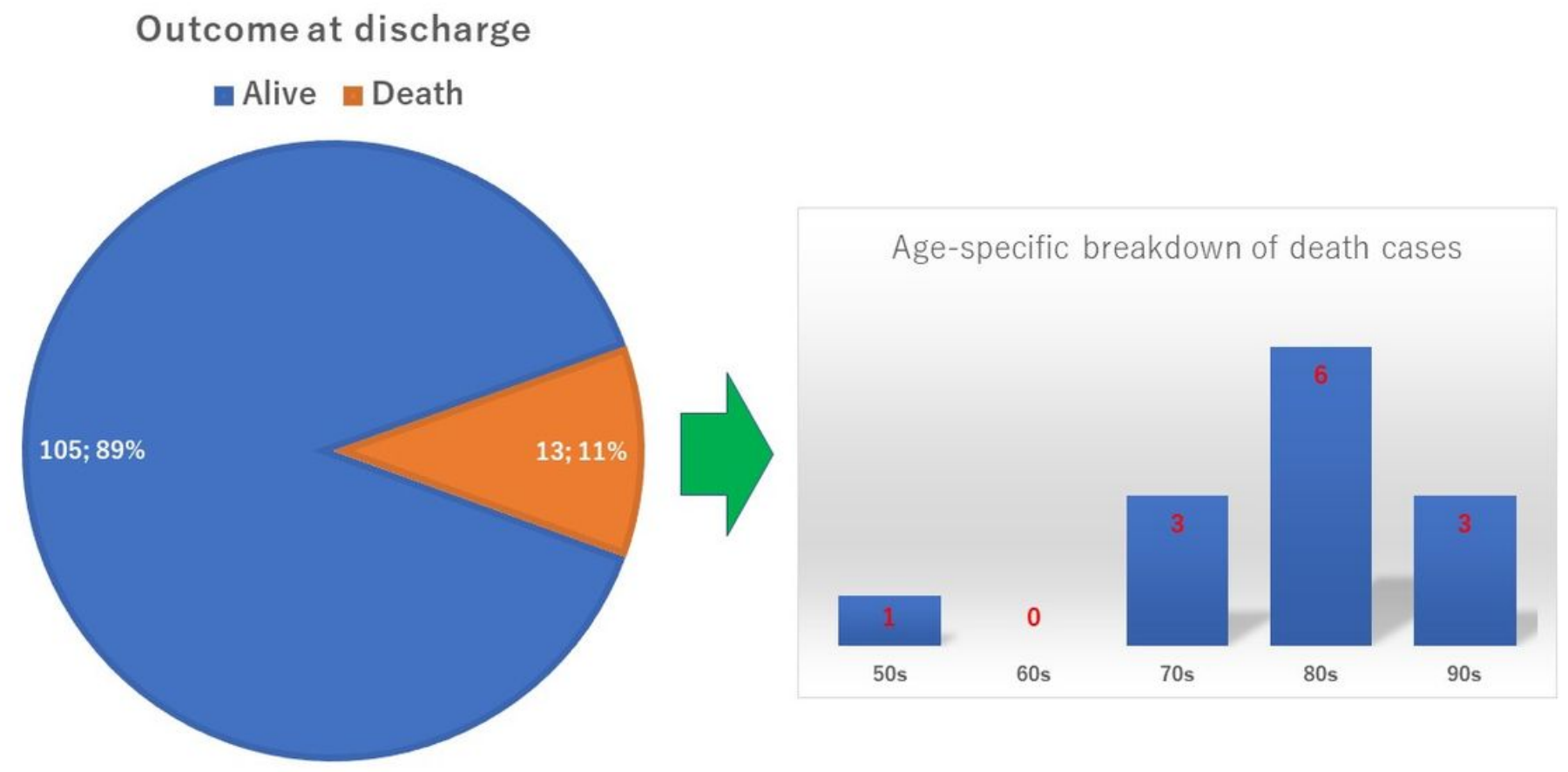
Figure 2

Outcome at discharge. The circle graph shows the percentages of alive and death cases. The bar graph shows the age-specific breakdown of the death cases.

\section{Duration of hospitalization}
Days
$\square$ Age $<80 \backsim$ Age $80 \leq$



\section{Figure 3}

Duration of the hospitalization. Orange and grey box plots show the mean duration of hospitalization in patients aged $<80$ years and those aged $\geq 80$ years, respectively. 